\title{
TRP-2 mediates coat color pigmentation in sheep skin
}

\author{
LINLI XUE ${ }^{1,2}$, YANAN LI $^{1}$, BINGLING ZHAO ${ }^{1}$, TIANZHI CHEN ${ }^{1}$, YANJUN DONG ${ }^{3}$, \\ RUIWEN FAN $^{1}$, JINGWEI LI ${ }^{1}$, HAIDONG WANG ${ }^{1}$ and XIAOYAN HE ${ }^{1}$
}

${ }^{1}$ College of Animal Science and Veterinary Medicine and ${ }^{2}$ College of Information, Shanxi Agricultural University,
Jinzhong, Shanxi 030801; ${ }^{3}$ College of Veterinary Medicine, China Agricultural University, Beijing 100193, P.R. China

Received July 25, 2017; Accepted December 14, 2017

DOI: $10.3892 / \mathrm{mmr} .2018 .8563$

\begin{abstract}
Tyrosinase-related protein 2 (TRP-2) is one of the most important members of the tyrosinase family, and is a key enzyme involved in melanin biosynthesis. In the present study, a skin transcriptome profile, immunohistochemistry, western blotting and reverse transcription-quantitative polymerase chain reaction were used to investigate TRP-2 expression in sheep with different coat colors, namely, black, white and black-white. TRP-2 was overexpressed in melanocytes in order to study the effect of TRP-2 on melanin production. Results revealed differing TRP-2 levels in sheep of different coat colors and in various parts of the coat with different colors in the same sheep. TRP-2 expression levels in dark-colored areas were significantly increased compared with light-colored areas in piebald sheep. TRP-2 overexpression may regulate melanogenesis and significantly increase melanogenesis associated transcription factor expression in vitro. Therefore, TRP-2 may affect melanin production in sheep, and different expression levels determine coat color. The results may provide novel approaches for developing therapeutic strategies for skin diseases associated with pigmentation disorders.
\end{abstract}

\section{Introduction}

The tyrosinase (TYR) gene family are derived from a common ancestor, subcategorized into TYR-related protein (TRP)-1 and TRP-2 during evolution. TRP-1 and TRP-2 are members of the family of dinuclear copper-binding proteins and contain two highly conserved copper-binding regions (1-3). TRP-1 and TRP-2 are similar to TYR, which also belongs to the TYR gene family, in terms of protein sequences, exhibiting the conserved structure of the TYR family $(4,5)$. TRP-2 participates in the synthesis of melanin, which interacts with various

Correspondence to: Dr Haidong Wang or Dr Xiaoyan He, College of Animal Science and Veterinary Medicine, Shanxi Agricultural University, 1 Mingxian South Road, Jinzhong, Shanxi 030801, P.R. China

E-mail: 13623541993@163.com

E-mail: 13753418018@126.com

Key words: coat color, melanocytes, sheep, tyrosinase-related protein 2 proteins and is present in human and animal skin and hair, in addition to the bark of certain plants and insect shells. In animals with woolly coats, TRP-2 may serve an important role in the early stages of TYR catalytic activity in melanin formation and is one of the key enzymes involved in melanin synthesis. For certain diseases, including albinism and vitiligo, TRP-2 exhibits important research significance (6).

TRP-2 contains at least eight exons and seven introns, and has a gene length of $60 \mathrm{~kb}$. All of the eight exons code for the TRP-2 protein (7). The TRP-2 carboxyl-terminal and membrane structure domain serve a key role in melanosome production (8). Valverde et al (9) demonstrated that the molecular weight of TRP-2 is $75 \mathrm{kDa}$. Dopa red pigment compounds may be isomerized to the colorless eumelanin medium, 5,6-dihydroxyindole-2-carboxylic acid (DHICA), by TRP-2, and hence, TRP-2 has been named dopachrome tautomerase (Dct) (10). Of the relevant members of the family, TRP-2 directly participates in the regulation of melanin production and melanocyte growth, survival and function (11). TRP-2 also controls the proportion of DHICA and 5,6-dihydroxyindole in melanocytes, which are important proteins that regulate animal coat color.

Current studies of TRP-2 focus on humans, mice, and rabbits. In humans, TRP-2 is a cytoplasmic antigen expressed in skin melanocytes and melanoma cells and is associated in the pathogenesis of vitiligo. TRP-2 autoantibodies may immunoreact with either TRP-2 or TYR as cross antigens, forming a solid foundation for research on the mechanism and treatment of vitiligo. In mice, studies on TRP-2 focus on its effect on melanin formation and thus its role in color regulation. Research has focused on white-coated, black-eyed iris rabbits for TRP-2 gene cloning and analysis. In the present study, the association between TRP-2 expression was investigated in sheep skin and various coat colors and the function of TRP-2 was determined via deep sequencing of skin transcriptome, immunohistochemistry, reverse transcription-quantitative polymerase chain reaction (RT-qPCR), cell transfection and western blotting. This work may provide a theoretical basis for research on the underlying regulatory mechanisms of animal coat color.

\section{Materials and methods}

Experimental animals and sample collection. Sheep housing and care and skin sample collection for experimental 
use were conducted in accordance with the International Guiding Principles for Biomedical Research Involving Animals (12). The study was approved by the Experimental Animals Ethical Committee of Shanxi Agricultural University (Shanxi, China).

During the study phases, the studied sheep were kept in the following conditions: $12 \mathrm{~h}$ light/dark cycle ratio, $25 \pm 2^{\circ} \mathrm{C}$, $50 \pm 5 \%$ humidity and acceptable ventilation conditions. In all stages, food and water were supplied freely to sheep. Following shaving and disinfection, nine Dorset sheep (Shanxi Hunyuan Sheep Yard, Jinzhong, China) were subjected to a skin biopsy using cutisectors. The sheep were all 1-year-old males and consisted of three sheep for each of the black, white and black-white coat colors. Every effort was made to minimize the suffering and number of animals used in the study. A total of 3 biopsies were collected from each of the black and white sheep, whereas three from dark-colored areas and three from light-colored areas were obtained in black-white sheep. A single sample was used for deep sequencing of skin transcriptome at Beijing Genomics Institute (Guangdong, China), one for RNA isolation and the last was fixed in $10 \%$ formalin overnight at $4^{\circ} \mathrm{C}$, processed, embedded in paraffin and sectioned at $5 \mu \mathrm{m}$ thickness.

RNA preparation, PCR and RT-qPCR. Total RNA was isolated from 12 sheep skin samples using TRIzol ${ }^{\circledR}$ reagent (Invitrogen; Thermo Fisher Scientific, Inc. Waltham, MA, USA). Total RNA concentrations were determined using a NanoDrop 2000 spectrophotometer (NanoDrop Technologies; Thermo Fisher Scientific, Inc., Pittsburgh, PA, USA), and electrophoresis performed using an $0.8 \%$ agarose gel to confirm the integrity of total RNA. Complementary strand cDNA was synthesized following the instructions of the reverse transcription kit (cat no. RR014A; Takara Biotechnology, Co., Ltd., Dalian, China). Following completion of RT, the samples were stored at $-20^{\circ} \mathrm{C}$ for later use. PCR was performed using these specific primers (Table I) and an appropriate amount of cDNA $(5 \mathrm{ng} / \mu \mathrm{l})$ as the template. Conditions of the PCR were as follows: Preheating at $95^{\circ} \mathrm{C}$ for $5 \mathrm{~min}$, followed by 35 cycles of shuttle heating at $95^{\circ} \mathrm{C}$ for $30 \mathrm{sec}$, annealing temperature at $60^{\circ} \mathrm{C}$ for $30 \mathrm{sec}$ and extending temperature at $72^{\circ} \mathrm{C}$ for $20 \mathrm{sec}$. The PCR products were detected by $1 \%$ agarose gel electrophoresis: After running at $220 \mathrm{~V}$ for $10 \mathrm{~min}$.

The levels of gene mRNA were measured using SYBR ${ }^{\circledR}$ Premix Ex TaqTMII (Tli RNaseH Plus, cat no. DRR081A; Takara Biotechnology, Co., Ltd.). The cDNA synthesis for RT-qPCR analysis of TRP-2 expression in sheep skin was performed using the NCode mRNA q-RT-PCR kit, according to the manufacturer's protocol (Invitrogen; Thermo Fisher Scientific, Inc.). In brief, total RNA was polyadenylated using poly A polymerase and adenosine triphosphate. Complementary DNA was synthesized using the NCode universal reverse primer, and RT-qPCR was performed using a TRP-2 primer. All reactions were performed in triplicate on the Stratagene Mx3005P Real-Time QPCR system. Conditions for RT-qPCR were as follows: Preheating at $95^{\circ} \mathrm{C}$ for $5 \mathrm{~min}$, followed by 40 cycles of shuttle heating at $95^{\circ} \mathrm{C}$ for $30 \mathrm{sec}$, annealing temperature at $60^{\circ} \mathrm{C}$ for $30 \mathrm{sec}$, extending temperature at $72^{\circ} \mathrm{C}$ for $20 \mathrm{sec}$. Quantification of TRP-2 transcriptional abundance was compared using the $2^{-\Delta \Delta \mathrm{Cq}}$ cyclic thresholding method (13), and the abundance of TRP-2 relative to $18 \mathrm{~S}$ was determined. RT-qPCR was performed as previously described (14-17). All primer sequences are listed in Table I.

Immunohistochemical analysis. The optimal conditions for the immunohistochemistry procedure were previously described (18-20). The present study used the streptavidin-biotin-enzyme complex (SABC) system and peroxidase (Biomeda staining kit; Dako, Agilent Technologies, Inc., Santa Clara, CA, USA), which is an indirect immunohistochemistry method using a streptavidin-biotin complex that allows signal amplification. Free-floating sections were washed in $0.1 \mathrm{M}$ PBS and incubated in $3 \%$ hydrogen peroxide for $15 \mathrm{~min}$ at room temperature to block the activity of endogenous peroxidase. Following washing with $0.1 \mathrm{M}$ PBS, the sections were boiled in $0.01 \mathrm{M}$ citric acid for $10 \mathrm{~min}$, followed by a $20 \mathrm{~min}$ immersion in PBS containing 5\% bull serum albumin (cat no. C500626; Sangon Biotech, Co., Ltd., Shanghai, China) at $37^{\circ} \mathrm{C}$. The sections were incubated overnight at $4^{\circ} \mathrm{C}$ with anti-TRP-2 (Dct H-150; cat no. sc-25544; 1:500; Santa Cruz Biotechnology, Inc., Dallas, TX, USA) and then at room temperature for $0.5 \mathrm{~h}$. Following three washes in $0.1 \mathrm{M} \mathrm{PBS}$ for $5 \mathrm{~min}$ each, the sections were incubated with biotinylated anti-rabbit IgG as a secondary antibody (cat no. BA1003; 1:100; Wuhan Boster Biological Technology, Ltd., Wuhan, China) at $37^{\circ} \mathrm{C}$ for 20-30 min. Following washing with PBS, the sections were incubated with avidin-biotin-peroxidase (1:200; SABC Elite; Wuhan Boster Biological Technology, Ltd.). The products of the immunoreaction were visualized by incubating the sections in a staining solution containing $0.04 \%$ 3,3'-diaminobenzidine, $0.06 \%$ hydrogen peroxide and $0.06 \%$ nickel sulfate at room temperature for 5-10 min. Nuclei were counterstained with hematoxylin at room temperature for $5 \mathrm{~min}$, and the sections were observed under a light microscope (DM3000 b; Leica Microsystems GmBH, Wetzlar, Germany). For the negative controls, PBS was substituted for the primary antibody (21).

Optical density of TRP-2 collection. A total of 15 immunohistological sections were prepared for each coat color. Using the image analysis system Image-pro plus version 6. 0 (Media Cybernetics, Inc., Rockville, MD, USA) to check the staining intensity: Objects were counted and characterized using manual and automatic measurement tools, and optical density; objects of interest were noted and density parameters, negative staining $=0$, positive staining $=1$, were employed for analysis. Three positive TRP-2 data were collected from melanocytes for each section and were used for statistical analysis.

Expression vector construction for TRP-2. An oligonucleotide sequence corresponding to the TRP-2 sequence was synthesized and ligated into a mammalian expression vector, $\mathrm{pLV}$. ExBi.P/Puro-CMV-eGFP-MCS-TYP2 by Cyagen Biosciences, Inc. (Guangzhou, China). The vector contained a cytomegalovirus promoter, which drives the expression of TRP-2 and green fluorescent protein (GFP). The TRP-2 sequence in the constructs was identical to the endogenous sequence. It was demonstrated that this plasmid was capable of simultaneously expressing TRP-2 and GFP in the present study. The GFP expression unit was used to monitor TRP-2 expression. The TRP-2 construct was transformed into Escherichia coli DH5 $\alpha$ (cat. no. CW0808; CW Biotech, Beijing, China) and 
Table I. Primer sequences.

\begin{tabular}{clc}
\hline Primer & \multicolumn{1}{c}{ Sequence } & Size \\
\hline TRP-2 & & \\
F1 & GGTTCTAAAGCCATGAGCCCT & $1586 \mathrm{bp}$ \\
R1 & CTAGAGCAAGGCGTGAGCAT & \\
F2 & GTCCTTCGCTTTGCCCTACT & $175 \mathrm{bp}$ \\
R2 & GACTCGGCGGTTGTAGTCAT & \\
18S & & \\
F & AGTCCCTGCCCTTTGTACACA & $198 \mathrm{bp}$ \\
R & TTATTGCTTAAGAATACGCGTAG & \\
\hline
\end{tabular}

TRP-2, tyrosinase-related protein 2; F, forward; R, reverse.

the positive colonies were verified by sequencing (Beijing Genomics Institute, Beijing, China).

Culture of sheep melanocytes and transfection of TRP-2. The skin of newborn sheep was removed using sterile techniques and digested with $0.25 \%$ dispase II (cat no. 17105041; Thermo Fisher Scientific, Inc.) at $4^{\circ} \mathrm{C}$ for $12-14 \mathrm{~h}$. The epidermis was then separated from the dermis using scalpel and forceps, cut into sections, and treated with $0.05 \%$ trypsin- $0.01 \%$ EDTA at $37^{\circ} \mathrm{C}$ for $10 \mathrm{~min}$. The melanocytes were seeded $\left(4.5 \times 10^{6}\right.$ cells $\left./ \mathrm{ml}\right)$ in a melanocyte basal medium supplemented with $0.2 \mu \mathrm{g} / \mathrm{ml}$ cholera toxin (Sigma-Aldrich; Merck KGaA, Darmstadt, Germany), $2.5 \mu \mathrm{g} / \mathrm{ml}$ fungizone (cat. no. V900919-1G; Sigma-Aldrich; Merck KGaA), $0.05 \mathrm{mg} / \mathrm{ml}$ gentamicin (cat. no. G1272; Sigma-Aldrich; Merck KGaA), $0.5 \mu \mathrm{g} / \mathrm{ml}$ hydrocortisone (cat. no. A610506; Sangon Biotech, Co., Ltd.), $50 \mu \mathrm{g} / \mathrm{ml}$ bovine pituitary extract (cat. no. 13028014; Invitrogen; Thermo Fisher Scientific, Inc.), $1 \mathrm{ng} / \mathrm{ml}$ basic fibroblast growth factor (cat. no. 13256029; Thermo Fisher Scientific, Inc.), 10 ng/ml tissue plasminogen activator (cat no. T0831; Sigma-Aldrich; Merck KGaA) and $5 \mu \mathrm{g} / \mathrm{ml}$ insulin (cat. no. I8040; Beijing Solarbio Science \& Technology Co., Ltd., Beijing, China). The cell culture plate was coated with $0.01 \%$ rat tail collagen (cat. no. C7661; Sigma-Aldrich; Merck KGaA). Vector-TRP-2 and empty vector (pLV.ExBi.P/Puro-CMV-eGFP-MCS, Cyagen Biosciences, Inc.) transfections were performed at $60-80 \%$ cell confluence, in accordance with the operation manual of the kit (cat. no. 11668027; Thermo Fisher Scientific, Inc.); normal untransfected melanocytes served as a control. The transfection reagent was Lipofectamine ${ }^{\circledR}$ and $3 \mathrm{ug} / \mathrm{ml}$ of each vector was used for transfection. Following $12 \mathrm{~h}$ of culture at $37^{\circ} \mathrm{C}$, the medium was replenished and the cells were observed under an inverted microscope (Leica Microsystems, Inc., Germany). The extracted cell Total RNA and proteins were subjected to RT-qPCR and western blot analysis, respectively.

Western blotting. Total protein was extracted from melanocytes by using a total protein extraction radioimmunoprecipitation assay lysis buffer (cat. no. CW23345; CW Biotech). Protein concentrations were measured using an Enhanced Bicinchoninic Acid Protein Assay kit (cat. no. P0009, Beyotime Institute of Biotechnology, Haimen, China) using pure bovine serum albumin (cat. no. ST023-50 g; Beyotime
Institute of Biotechnology) as a reference. Extracts were denatured at $95^{\circ} \mathrm{C}$ for $5 \mathrm{~min}$. For $10 \%$ SDS-PAGE, $100 \mu \mathrm{g}$ total protein sample was deposited in each well. The separated protein was transferred to a nitrocellulose membrane, which was blocked with $5 \%$ skim milk at room temperature for $1 \mathrm{~h}$ and then incubated with a TRP-2 mouse monoclonal antibody (TRP2 C9; 1:500; cat. no. sc-74439; Santa Cruz Biotechnology, Inc.) and a GAPDH rabbit polyclonal antibody as an internal reference $(1: 3,000$; cat no. sc-25778; Santa Cruz Biotechnology, Inc.) at $4^{\circ} \mathrm{C}$ overnight. To reveal TRP-2 interference in melanogenesis associated transcription factor (MITF), which is an important transcription factor located upstream of TRP-2 involved in the melanogenesis pathway that is associated with pigmentation, MITF protein expression levels (total and phosphorylated protein) were measured by incubating with a MITF mouse monoclonal antibody (MITF 21D1418; 1:200; cat. no. sc-52938; Santa Cruz Biotechnology, Inc.) at $4^{\circ} \mathrm{C}$ overnight. On the second day, the membrane was transferred to room temperature for $30 \mathrm{~min}$ and washed three times In TBST with $0.1 \%$ Tween-20 for $10 \mathrm{~min}$ each. Horse radish peroxidase-conjugated anti-mouse $\operatorname{IgG}(1: 10,000$; cat. no. BA1001; Wuhan Boster Biological Technology, Ltd.) was added to cover the membrane and incubated at $37^{\circ} \mathrm{C}$ with horizontal shaking for $1 \mathrm{~h}$. Following washing six times with TBST for 5 min each, the membrane was developed using the highly sensitive, enhanced chemiluminescence solution (cat. no. CW00495; CW Biotech) consisting of solutions A and B at $1: 1$ ratio followed by film exposure. The resulting image was scanned. The TRP-2, MITF and GAPDH immunoblot results were analyzed using the Image-ProPlus version 6.0 software (Media Cybernetics, Inc., Rockville, MD, USA) to measure the area and gray value of each target band. Semi-quantitative analysis was performed by comparing the target protein and the internal reference using the following equations: Protein content=area of the band $\mathrm{x}$ average gray scale; semi-quantitative target protein content=target protein content/GAPDH protein content. All data were presented as mean \pm standard error. Univariate analysis of variance and a Tukey's post hoc test were performed using SPSS software, version 19.0 (IBM SPSS, Armonk, NY, USA), and P<0.05 was considered to indicate a statistically significant difference.

Determination of melanin content. Constitutive and TRP-2 over-expressive melanin contents were compared on the third day following transfection. Melanin content was determined as previously described (22). Melanocytes were harvested, rinsed with PBS, and counted. Melanin was solubilized in $0.2 \mathrm{~mol} / \mathrm{l}$ $\mathrm{NaOH}$, resulting in a cell count of $10^{6}$ cells $/ \mathrm{ml}$, and measured spectrophotometrically at an absorbance of $475 \mathrm{~nm}$ against a standard curve of known synthetic melanin (Sigma-Aldrich; Merck KgaA) concentrations. Melanin content was expressed as $\mu \mathrm{g} / 10^{6}$ cells. All experiments were performed in triplicate.

Statistical analysis. All experiments were performed in triplicate. Data are presented as the mean \pm standard deviation. Differences in the protein abundance of TRP-2 in sheep skin samples of various coat colors were determined by univariate analysis of variance, followed by a Tukey's post hoc test using SPSS software, version 19.0 (IBM SPSS). $\mathrm{P}<0.05$ was considered to indicate a statistically significant difference. 
Table II. Differentially expressed known coat color genes in black vs. white sheep skin.

\begin{tabular}{llcl}
\hline Symbol & \multicolumn{1}{c}{ Gene name } & $\begin{array}{c}\text { Differential } \\
\text { expression }\end{array}$ & \\
\hline Edn3 & Endothelin 3 & 4.903 & Function \\
Dct $($ Trp -2$)$ & Dopachrome tautomerase & 6.023 & Melanosomal enzyme \\
Gpnmb & Glycoprotein NMB & 4.281 & Apparent melanosomal component \\
Matp & Membrane-associated transporter protein & 32.75 & Apparent transporter \\
Rab38 & RAB38, member RAS oncogene family & 2.206 & Targeting of Trp1 \\
Si & Silver & 25.2 & Melanosome matrix \\
Tyr & TYR & 61.79 & Melanosomal enzyme \\
Trp-1 & TYR-related protein 1 & 284.17 & Melanosomal protein \\
Mlph & Melanophilin & 27.75 & Melanosome transport \\
Ggt1 & Glutamyltranspeptidase 1 & 5.747 & Glutathione metabolism (pheomelanin synthesis) \\
Mc1r & Melanocortin 1 receptor & 9.315 & Eumelanin/pheomelanin switch (among others) \\
Mgrn1 & Mahogunin, ring finger 1 & 2.814 & Melanin color, CNS role. E3 ubiquitin ligase
\end{tabular}

\section{Results}

Transcriptome sequencing and analysis of black and white sheep skins. A total of 74,533 and 90,006 unigenes were assembled from the reads obtained from black and white sheep skin, respectively. Genes encoding for the ribosomal proteins and keratin-associated proteins were the most highly expressed. A total of 2,235 known genes were differentially expressed in black versus white sheep skin, with 479 genes upregulated and 1,756 genes downregulated. A total of 845 novel genes were differentially expressed in black sheep skin compared with white sheep skin, 107 of which were upregulated, including 2 highly expressed genes exclusively expressed in black sheep skin, whereas 738 were downregulated. A total of 49 known coat color genes were expressed in sheep skin, 12 of which revealed high expression in black sheep skin (Table II). A number of these upregulated genes, including TRP-2 (Dct), membrane-associated transporter protein, TYR and TRP-1 are members of melanosome components and their precursor ontology category.

TRP-2 mRNA expression. In the skins of white sheep, light-colored and dark-colored regions of piebald sheep, and black sheep, the expression levels of the TRP-2 gene following PCR amplification was determined, by $0.8 \%$ gel electrophoresis. The results revealed that the four groups produced TRP-2 bands without any nonspecific background. TRP-2 was 1,586 bp (representative data shown, Fig. 1A). Following sequencing, 4 TRP-2 sequences consistently aligned with the NCBIpublished sequences from Bos taurus, Capra hircus, Camelus bactrianus, Sus scrofa, Homo sapiens and Balaenoptera acutorostrata scammoni via Basic Local Alignment Search Tool, demonstrating alignment scores of 100, 100, 98, 99, 99 and $99 \%$, respectively (https://blast.ncbi.nlm.nih.gov/Blast .cgi?PROGRAM=blastn\&PAGE_TYPE=BlastSearch\&LINK_ LOC=blasthome). Following evaluation of mRNA integrity, the RT-qPCR amplification curve and melting curve revealed optimal results and the target gene, TRP-2, and reference genes did not produce nonspecific amplification or primer dimers (data not shown). The results of the data analysis demonstrated that TRP-2 mRNA expression levels in black sheep, dark-colored regions of piebald sheep and light-colored regions in piebald sheep was $1.75,1.67$ and 0.94 times increased compared with white sheep (Fig. 1B). TRP-2 mRNA expression levels in black sheep and dark-colored regions in piebald sheep were significantly increased compared white sheep and light-colored regions in piebald sheep (Fig. 1B; $\mathrm{P}<0.05$ ). However, the difference in the expression levels of black sheep and dark-colored regions was not significant (Fig. 1B). The difference in expression levels between white sheep and light-colored regions in piebald sheep was also not significant (Fig. 1B). These findings suggested that differences in coat color of sheep may be associated with the TRP-2 mutation.

Immunolocalization and TRP-2 expression levels in sheep of various coat colors. Immunohistochemical assays were performed to determine the localization of TRP-2 in sheep coats of various colors. TRP-2 was expressed in the epidermis, inner root sheath and dermal papilla of skins (representative data shown, Fig. 2A-D). Different expression levels of TRP-2 were observed in various coat colors and in the same sheep at regions of different coat colors. In white sheep, TRP-2 staining was weakly positive (Fig. 2A), whereas that in black was strongly positive, particularly in the dermal papilla and inner root sheath melanocytes (Fig. 2C). TRP-2 staining intensity was similar between single-colored sheep and those with various colored regions (Fig. 2A-D). TRP-2 expression levels were lowest in white-coated sheep and highest in black-coated sheep (Fig. 2A and C). Furthermore, the levels of TRP-2 in dark-colored areas were significantly higher compared with light-colored areas in piebald sheep (Fig. 2D and B). Melanin synthesis was associated with TRP-2 expression. The control group demonstrated no positive staining (Fig. 2E-H).

Optical density analysis of the immunohistochemical results. Average optical density values for TRP-2 were $2.13 \pm 0.14$, $2.04 \pm 0.08,1.21 \pm 0.06$ and $1.00 \pm 0.06$ in black skin, black dots, white dots and white skin, respectively (Fig. 3). These values 

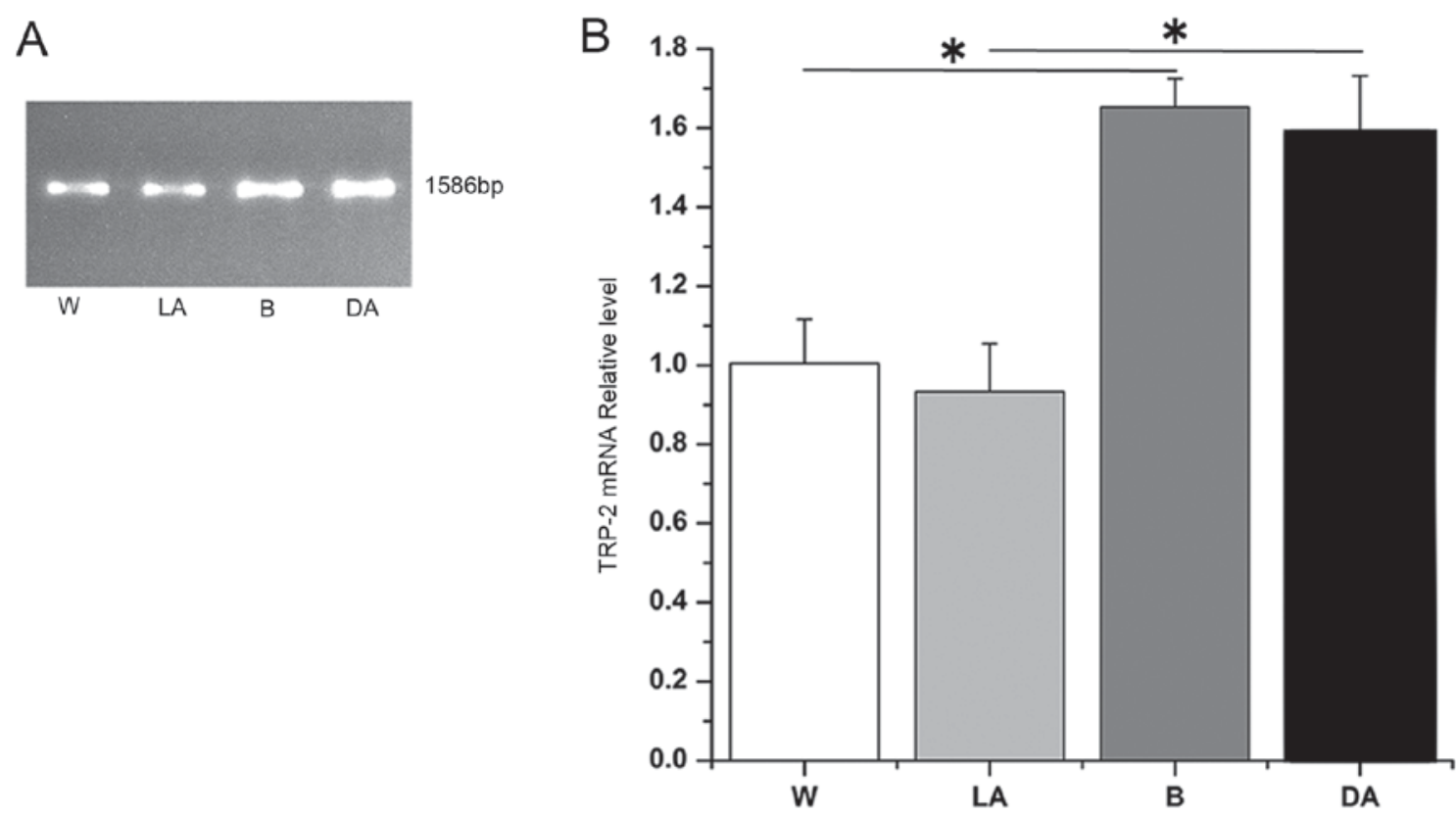

Figure 1. TRP-2 mRNA expression levels in sheep skin of various coat colors. (A) Representative image and (B) quantification of TRP-2 mRNA expression levels detected by quantitative polymerase chain reaction. " $\mathrm{P}<0.05$. W, white sheep; LA, light-colored areas in black-white sheep; B, black sheep; DA, dark-colored areas and in black-white sheep. TRP-2, tyrosinase-related protein 2.
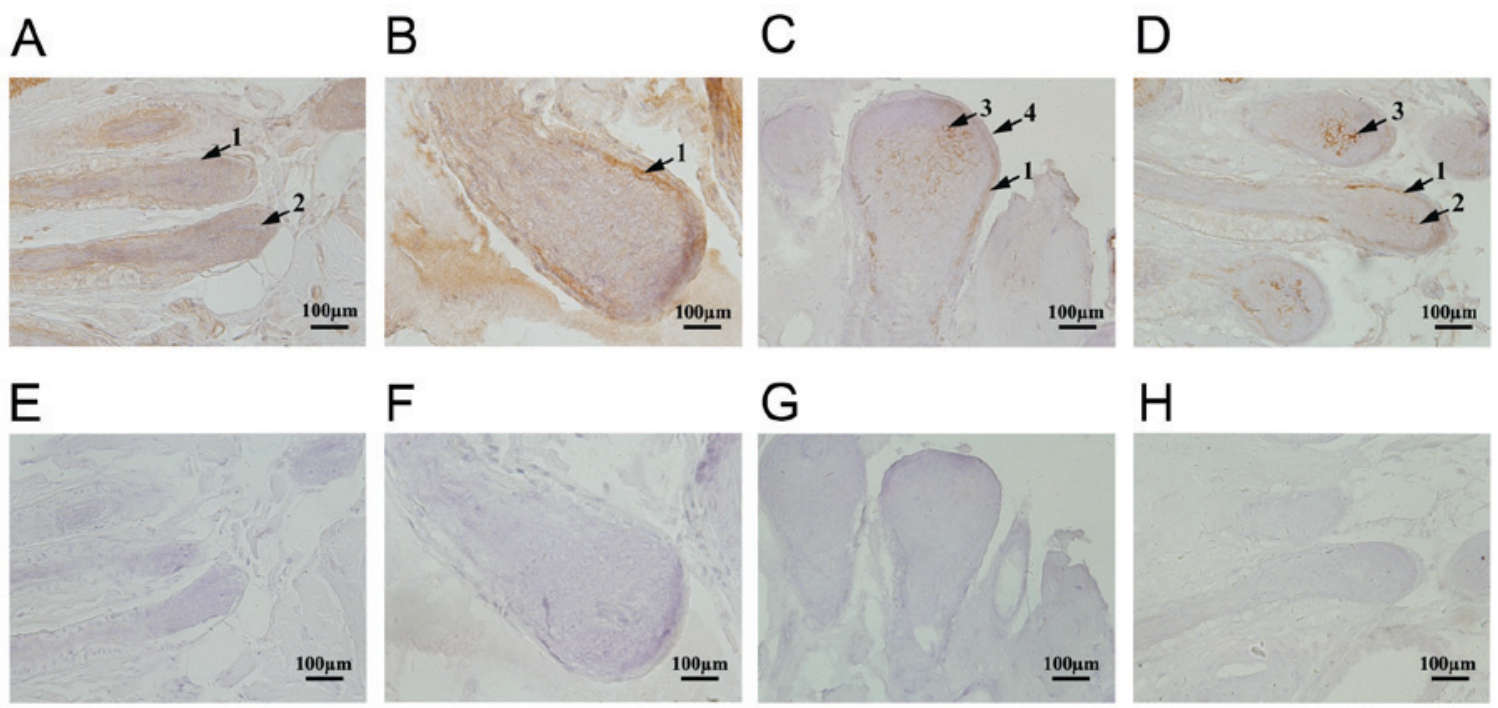

Figure 2. Immunohistochemistry results of TRP-2 in sheep with different coat colors. (A) White sheep coat TRP-2 positive group. (B) Light-colored areas in black-white sheep TRP-2 positive group. (C) Black sheep coat TRP-2 positive group. (D) Dark-colored areas in black-white sheep TRP-2 positive group. (E) White sheep coat TRP-2 negative control group. (F) Light-colored areas in black-white sheep TRP-2 negative control group. (G) Black sheep coat TRP-2 negative control group. (H) Dark-colored areas in black-white sheep TRP-2 negative control group. Scale bar, $100 \mu \mathrm{m}$. TRP-2, tyrosinase-related protein 2. Numbers denote: 1-inner root sheath; 2-dermal papilla; 3-hair follicle matrix; 4-outer root sheath.

indicated that TRP-2 was expressed at 1.76- and 2.13-fold increased levels in black skin versus light-colored areas in piebald skin and white skin and 1.68- and 2.04-fold higher in dark-colored areas versus light-colored areas in piebald skin and white skin, respectively. No significant difference in TRP-2 expression between black skin and dark-colored areas in piebald skin was observed (Fig. 3).

Detection of melanin following transfection of sheep melanocytes with TRP-2. To determine the effects of TRP-2, TRP-2 was overexpressed by transfecting logarithmic-phase primary melanocytes with the TRP-2 plasmid. The melanocytes exhibited good growth conditions, with the morphology revealing polygon and dendritic protrusions. Following transfection, melanocytes demonstrated normal growth status and high transfection efficiency (Fig. 4A). Cells transfected with the pLV.ExBi.P/Puro-CMV- eGFP-MCS-TYP2 construct demonstrated a 5-fold higher expression of TRP-2 compared with cells transfected with the pLV.ExBi.P/Puro-CMV-eGFP-MCS vector control (Fig. 4B). The melanin content was $6.54 \pm 0.24$, $6.19 \pm 0.54$ and $13.08 \pm 0.69 \mu \mathrm{g} / 10^{6}$ cells in the control, vector without TRP-2 and vector-TRP-2 group, respectively, 


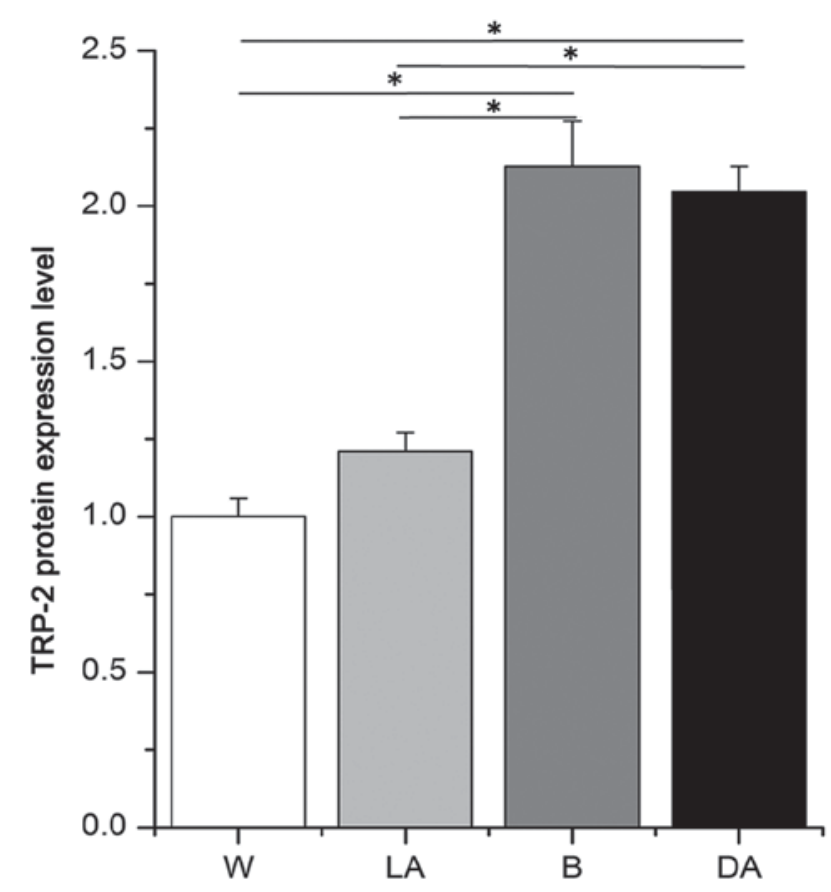

Figure 3. Relative protein expression levels of the TRP-2 protein in sheep of different coat colors, as measured by immunohistochemistry staining. W, white sheep; LA, light-colored areas in black-white sheep; B, black sheep; DA, dark-colored areas in black-white sheep. ${ }^{*} \mathrm{P}<0.05$. TRP-2, tyrosinase-related protein 2 .

suggesting that the overexpression of TRP-2 produced more melanin compared with the other groups (Fig. 4C). These data support the hypothesis that TRP-2 overexpression may enhance melanin content and influence coat color synthesis. In addition, western blotting results demonstrated that TRP-2 protein levels were significantly increased 1.38 -fold in cells overexpressing TRP-2 compared with those transfected with the vector control (Fig. 5A).

It was demonstrated that MITF was significantly increased in the TYP2-overexpressing cells compared with the control group (Fig. 5A and B), suggesting that TRP-2 regulates expression of MITF.

\section{Discussion}

In mammals, the development of skin accessory organs, including hair follicles and hair shafts, involves cycles of growth (anagen), regression (catagen) and rest (telogen) (23). Hair follicles have a complicated shape and structure and a three-dimensional conformation. They control hair cyclical growth and renew themselves. Once hair follicles are formed in the embryo, dermal papilla and the first half of the hair follicle do not alter further following birth, whereas the other hair follicles, along with stromal cells and nutrients, undergo cyclic alterations (24). In the human mature follicle, dopamine-positive melanocytes are easily detected around the hair bulb base layer and dermal papilla, and moderately differentiated melanocytes may be detected in the base layer of sebaceous glands. Dopamine-negative melanocytes that cannot produce melanin distribute in the middle of the outer root sheath or base, whereas others are distributed in the peripheral region of the hair bulb and the adjacent matrix. It has previously been demonstrated that immature melanoblasts may exist in the mature follicles, and no dopa oxidase activity occurs in the absence of pigmented hair follicles; however, in certain dopamine-negative melanocytes, a small amount of TYR is detected. Similarly, the expression of mast/stem cell growth factor receptor, B-cell lymphoma 2 and non-pigmented melanocytes also exist in hair follicles which do not express TRP-1 and TRP-2 (25).

The present study revealed that TRP-2 was expressed in various coat colors of adult sheep skins. TRP-2 existed in melanocytes at the root sheath in white sheep and in light-colored areas in piebald sheep. TRP-2 was expressed in melanocytes of dermal papilla and hair root sheaths in black sheep and dark-colored areas in piebald sheep, which was consistent with previous study (26). These results suggested that melanocytes of hair follicles, dermal papilla and root sheaths in black sheep and back-colored regions in piebald sheep may produce melanin. On the contrary, the cells around hair root sheath in white sheep and light-colored regions may be dopa-negative melanocytes, which are not involved in melanin production.

The synthesis, transport, and distribution of melanin in the mammalian skin, hair follicles and eyes results in the appearance of various colors $(16,27)$. In melanocytes, specific organelles called melanosomes produce melanin. TYR serves a vital role in this process and is responsible for catalyzing three different reactions of melanin: Catalysis to dopa, oxidation to dopaquinone, hydroxylation to dihydroxyindole. In addition, TRP-2 participates in melanin synthesis in certain reactions: TRP-2, which possesses the activity of dopa pigment isomerase, catalyzes dopa pigments into DHICA (28). The present in vitro study suggested that overexpression of TRP-2 affects melanin production. In HeLa (29) and COS (26) cells, TRP-2 expression may lead to the extraction of dopa pigment from these cells into DHICA, which is the product of TRP-2 and may be used as substrates of TRP-1. TRP-2 is an important protein that affects the formation of melanin, and its expression and activity determines the speed of melanin production and yield. Tyrosine hydroxyl is converted to dopamine by TYR, whereas dobutamine is oxidized into dopaquinone and further into various derivatives. Melanin is a dopaquinone, indole-5 or 6 quinone and dopa pigment polymer. TRP-2 has become an important protein to study melanin traits and albinism.

TRP-2 shares $40 \%$ identity with TYR and is a useful marker for melanocyte differentiation. From dopaquinone, the eumelanin and pheomelanin pathways diverge. TRP-1 and TRP-2 are two crucial enzymes involved in eumelanogenesis. Pheomelanin is derived from conjugation by glutathione or thiol-containing cysteine. Therefore, pheomelanin is more photolabile and produces as by-products, hydrogen peroxide, hydroxyl radicals and superoxide, which result in further DNA damage. Individual melanocytes typically synthesize both eumelanins and pheomelanins, with the ratio of the two being determined by a balance of variables, including pigment enzyme expression and availability of tyrosine and sulfhydryl-containing reducing agents in the cells (30).

In the present study, it was demonstrated that the TRP-2 protein was expressed in all coat colors of sheep, and the 
A
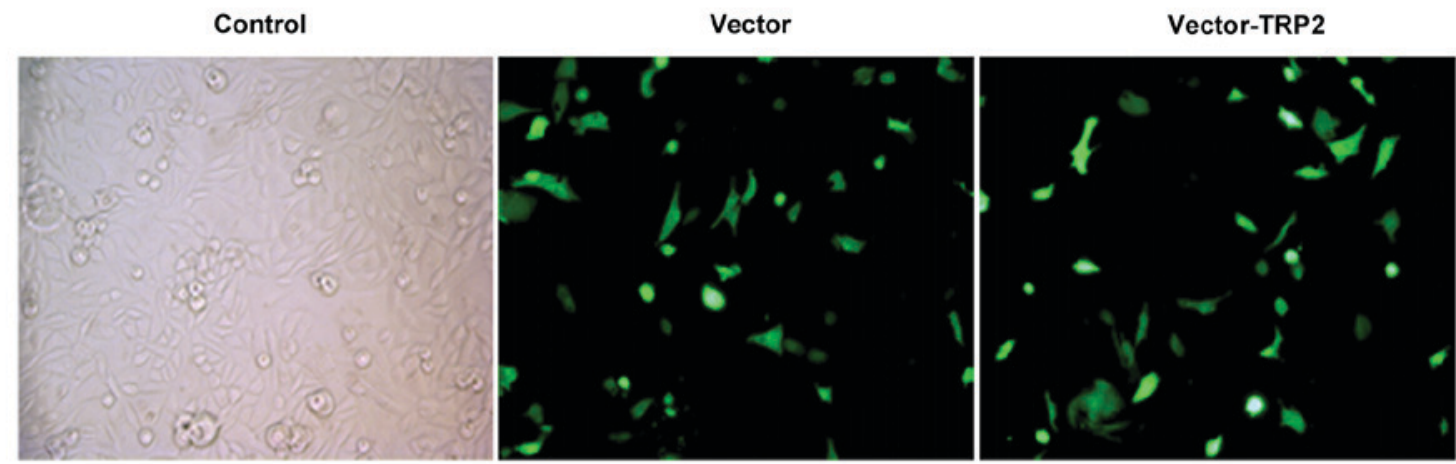

B

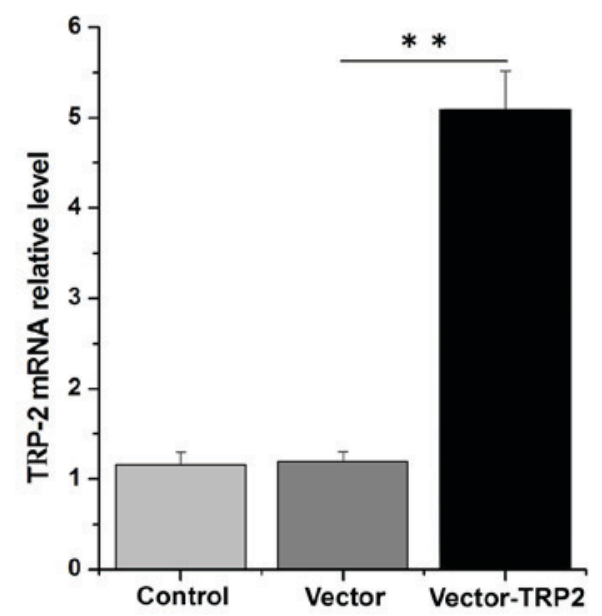

C

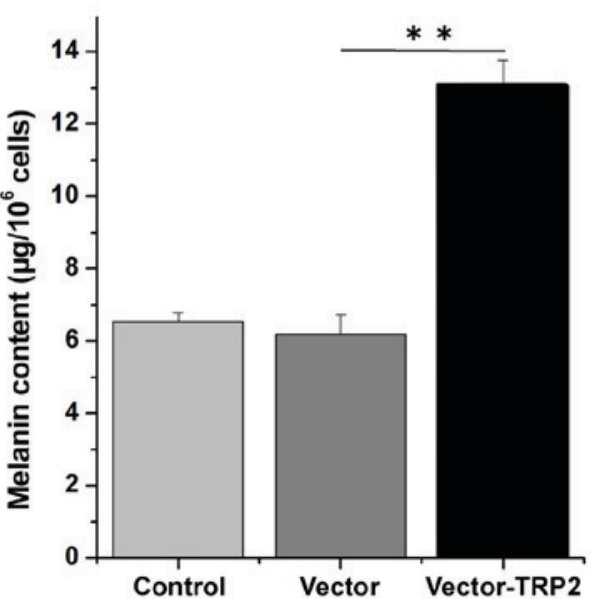

Figure 4. Melanin content following transfection of sheep melanocytes with a TRP-2-containing plasmid. (A) Cell morphology and TRP-2 transfection efficiency. (B) TRP-2 mRNA relative expression levels in melanocytes following transfection. (C) Alterations in melanin content in overexpressed melanocytes. TRP-2 overexpression resulted in increased melanin production. ${ }^{* *} \mathrm{P}<0.01$. Control, non-transfected melanoctye group; Vector, melanocyte transfection with pLV.ExBi.P/Puro-CMV-eGFP-MCS without TRP-2; Vector-TRP-2, melanoctye transfection with pLV.ExBi.P/Puro-CMV-eGFP- MCS-TYP2; TRP-2, tyrosinase-related protein 2 .

A

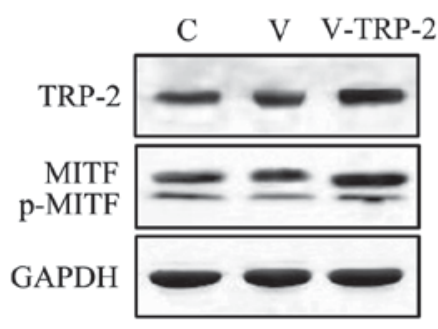

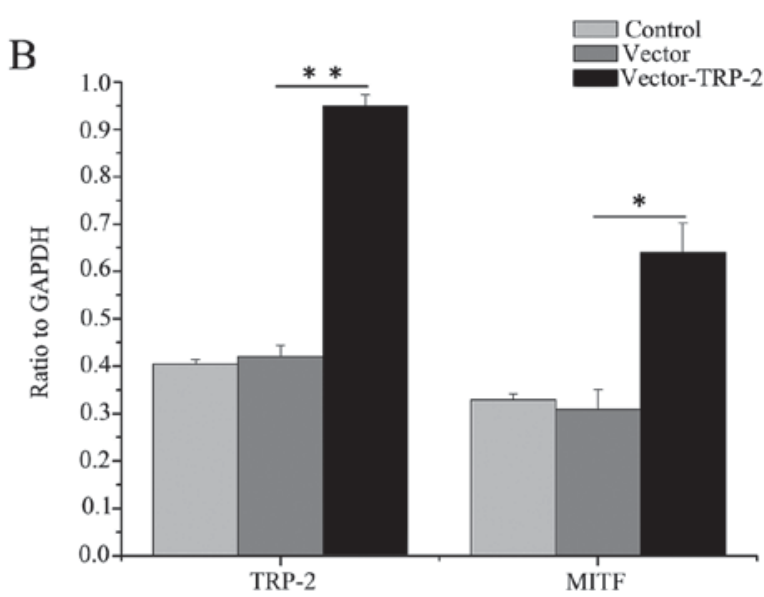

B

increased compared with white sheep and light-colored regions. Expression was most increased and decreased in sheep with expression levels of the TRP-2 protein in black sheep and dark-colored regions in piebald sheep were significantly

Figure 5. TRP-2 and MITF relative protein expression levels. (A) Representative image and (B) quantification of western blotting results revealed that TRP-2 and MITF proteins were significantly enhanced following TRP-2 overexpression. ${ }^{*} \mathrm{P}<0.05$ and ${ }^{* *} \mathrm{P}<0.01$. TRP-2, tyrosinase-related protein 2 ; MITF, melanogenesis associated transcription factor; C, control; V, vector; V-TRP2, vector-TRP2. 
black and white coats, respectively. TRP-2 expression quantity and pigment production or coat color formation were associated. However, there was a direct association between black or white coats of sheep and TRP-2 expression. In conclusion, TRP-2 was expressed in sheep of all coat colors. To a certain extent, TRP-2 expression levels reflected the numbers of mature melanocytes in sheep of various coat colors, indicating an important role for TRP-2 in the regulation of melanin production and coat color formation in sheep. Whether the expression of TRP-2 affects eumelanin and pheomelanin formation in different coat-colored sheep skins require further investigation. The findings of the present study may provide novel insights for clinical research into skin diseases associated with pigmentation disorders.

\section{Acknowledgements}

Not applicable.

\section{Funding}

The present study was sponsored by the National Natural Science Foundation of China (grant nos. 31772690 and 31302049), and the Science and Technology Innovation Foundations of Shanxi Colleges and Universities (grant no. 20171115).

\section{Availability of data and materials}

Not applicable.

\section{Authors' contributions}

HW and XH designed the study. LX, BZ, RF and YL conducted the studies and participated in data collection. TC and YD performed the statistical analysis and assisted in the study design. JL performed statistical analysis and helped draft the manuscript. All authors read and approved the final manuscript.

\section{Ethics approval and consent to participate}

The present study was approved by the Experimental Animals Ethical Committee of Shanxi Agricultural University (Shanxi, China). Experimental protocols were conducted in accordance with the International Guiding Principles for Biomedical Research Involving Animals (12).

\section{Consent for publication}

Not applicable.

\section{Competing interests}

The authors declare that they have no competing interests.

\section{References}

1. Iwata M, Corn T, Iwata S, Everett MA and Fuller BB: The relationship between tyrosinase activity and skin color in human Foreskins. J Invest Dermatol 95: 9, 1990.
2. Commo S, Gaillard OS Thibaut S and Bernard B: Absence of TRP-2 in melanogenic melanocytes of human hair. Pigment Cell Res 17: 488-497, 2004.

3. Pomerantz SH and Ances IG: Tyrosinase activity in human skin. Influence of race and age in newborns. J Clin Invest 55: 1127-1131, 1975

4. Zhu L, Cai YQ, Jue TU and Chen ML: Cloning and analysis of Trp1 and Trp2 genes from white hair black eyes rabbit iris. Acta Laborat Anim Scientia Sin 20: 43-48, 2012.

5. Hearing VJ: The melanosome: The perfect model for cellular responses to the environment. Pigment Cell Res 13 (Suppl 8): S23-S24, 2000

6. Zhu Y and Xu A: The research progress of vitiligo animal model. Int J Dermatol Venereal 39: 120-123, 2013.

7. del Marmol V, Ito S, Jackson I, Vachtenheim J, Berr P, Ghanem G, Morandini R, Wakamatsu K and Huez G: TRP-1 expression correlates with eumelanogenesis in human pigment cells in culture. FEBS Lett 327: 307-310, 1993.

8. Gao L, Dong CS, He XY, He JP, Geng JJ, Fan RW, Zhu ZW and You RL: Gene expression levels of alpaca tyrosinase gene family in individuals of different colors. Chin J Ani Veter Sci 39: 895-899, 2008 (In Chinese).

9. Valverde P, Healy E, Jackson I, Rees JL and Thody AJ: Variants of the melanocyte-stimulating hormone receptor gene are associated with red hair and fair skin in humans. Nat Genet 11: 328-330, 1995

10. Wang Q, Gao T, Li C, Shen Z and Li Q: Cloning and expression of human tyrosinase related protein-2 cDNA. J Fourth Military Med Univ 23: 629-632, 2002 (In Chinese).

11. Jiménez-Cervantes C, Solano F, Kobayashi T, Urabe K, Hearing VJ, Lozano JA and García-Borrón JC: A new enzymatic function in the melanogenic pathway. The 5,6-dihydroxyindole-2-carboxylic acid oxidase activity of tyrosinase-related protein-1 (TRP1). J Biol Chem 269: 17993-8000, 1994.

12. (CIOMS) International Guiding Principles for Biomedical Research Involving Animals. Altern Lab Anim 12: ii-, 1985.

13. Livak KJ and Schmittgen TD: Analysis of relative gene expression data using real-time quantitative PCR and the 2(-Delta Delta C(T)) method. Methods 25: 402-408, 2001.

14. Dong C, Wang H, Xue L, Dong Y, Yang L, Fan R, Yu X, Tian X, Ma S and Smith GW: Coat color determination by miR-137 mediated down-regulation of microphthalmia-associated transcription factor in a mouse model. RNA 1679-1686, 2012

15. Goswami S, Tarapore RS, Teslaa JJ, Grinblat Y, Setaluri V and Spiegelman VS: MicroRNA-340-mediated degradation of microphthalmia-associated transcription factor mRNA is inhibited by the coding region determinant-binding protein. J Biol Chem 285: 20532-20540, 2010.

16. Zhu Z, He J, Jia X, Jiang J, Bai R, Yu X, Lv L, Fan R, He X, Geng J, et al: MicroRNA-25 functions in regulation of pigmentation by targeting the transcription factor MITF in alpaca (Lama pacos) skin melanocytes. Domest Anim Endocrinol 38: 200-209, 2010.

17. Segura MF, Hanniford D, Menendez S, Reavie L, Zou X, Alvarez-Diaz S, Zakrzewski J, Blochin E, Rose A, Bogunovic D, et al: Aberrant miR-182 expression promotes melanoma metastasis by repressing FOXO3 and microphthalmiaassociated transcription factor. Proc Natl Acad Sci USA 106: 1814-1819, 2009.

18. Wang H, Dong Y, Chen W, Hei J and Dong C: Expression and localization of nerve growth factor (NGF) in the testis of alpaca (llama pacos). Folia Histochem Cytobiol 49: 55-61, 2011.

19. Tibary A and Vaughan J: Reproductive physiology and infertility in male South American camelids: A review and clinical observations. Small Ruminant Res 61: 283-298, 2006.

20. Yan YP, Dong CS, HE JP, Ren YH, He XY, Bai R and Xie JS: Expression and localization of transforming growth factor- $\beta 1$ in Alpaca testis. Chin J Animal Veter Sci 39: 97-102, 2008 (In Chinese).

21. Ayer-Lelievre C, Olson L, Ebendal T, Hallböök F and Persson H: Nerve growth factor mRNA and protein in the testis and epididymis of mouse and rat. Proc Natl Acad Sci USA 85: 2628-2632, 1988.

22. Chen T, Wang H, Liu Y, Zhao B, Zhao Y, Fan R, Wang P and Dong C: Ocular albinism type 1 regulates melanogenesis in mouse melanocytes. Int J Mol Sci 17: E1596, 2016.

23. Alonso L and Fuchs E: The hair cycle. J Cell Sci 119: 391-393, 2006.

24. Müller-RöverS,Handjiski B, van der Veen C,Eichmüller S,FoitzikK, McKay IA, Stenn KS and Paus R: A comprehensive guide for the accurate classification of murine hair follicles in distinct hair cycle stages. J Invest Dermatol 117: 3-15, 2001. 
25. Horikawa T, Norris DA, Johnson TW, Zekman T, Dunscomb N, Bennion SD, Jackson RL and Morelli JG: DOPA-negative melanocytes in the outer root sheath of human hair follicles express premelanosomal antigens but not a melanosomal antigen or the melanosome-associated glycoproteins tyrosinase, TRP-1, and TRP-2. J Invest Dermatol 106: 28-35, 1996.

26. Budd PS and Jackson IJ: Structure of the mouse tyrosinaserelated protein-2/dopachrome tautomerase (Tyrp2/Dct) gene and sequence of two novel slaty alleles. Genomics 29: 35-43, 1995.

27. De Luca M, D'Anna F, Bondanza S, Franzi AT and Cancedda R: Human epithelial cells induce human melanocyte growth in vitro but only skin keratinocytes regulate its proper differentiation in the absence of dermis. J Cell Biol 107: 1919-1926, 1988.

28. Winder AJ, Wittbjer A, Odh G, Rosengren E and Rorsman H: The mouse brown (b) locus protein functions as a dopachrome tautomerase. Pigment Cell Res 7: 305-310, 1994.
29. Yasumoto K, Yokoyama K, Takahashi K, Tomita Y and Shibahara S: Functional analysis of microphthalmia-associated transcription factor in pigment cell-specific transcription of the human tyrosinase family genes. J Biol Chem 272: 503-509, 1997.

30. Land EJ and Riley PA: Spontaneous redox reactions of dopaquinone and the balance between the eumelanic and phaeomelanic pathways. Pigment Cell Melanoma Res 13: 273-277, 2000.

This work is licensed under a Creative Commons Attribution-NonCommercial-NoDerivatives 4.0 International (CC BY-NC-ND 4.0) License. 